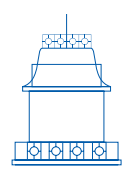

ARTÍCULOS

DE INVESTIGACIÓN

\title{
Consideraciones para una actualización del concepto de totalidad social con base en la obra de Georg Lukács*
}

\author{
Alejandro Nahuel Alzu \\ Facultad de Filosofía y Letras, Universidad de Buenos Aires, Buenos Aires, Argentina \\ E-mail: nalzu@filo.uba.ar \\ Recibido: 30 de octubre 2019 | Aprobado: 1 de mayo de 2020 \\ https://doi.org/10.17533/udea.ef.n62a05
}

\begin{abstract}
Resumen: En este artículo reviso las diversas elaboraciones del concepto de totalidad social que podemos encontrar en diferentes momentos de la obra del filósofo marxista Georg Lukács y destaco las concepciones de la praxis de los agentes sociales asociadas a cada una de ellas. Comienzo por analizar este concepto en la obra del joven Lukács, para luego presentar un radical cambio de postura entre las formulaciones que hallamos en Historia y conciencia de clase y Para una ontología del ser social. Mi argumento es que este cambio de perspectiva se asocia a la necesidad teórica de posibilitar una práctica subjetiva que no se encuentre indefectiblemente alienada, tal como parece desprenderse del modelo teórico de la obra lukácsiana de juventud. Finalmente concluyo que esta nueva concepción de la totalidad social habilita un nuevo tipo de práctica emancipatoria, alejada de los dogmatismos partidarios que caracterizaron a buena parte del socialismo del siglo XX.
\end{abstract}

Palabras clave: totalidad, reificación, alienación, capitalismo, marxismo

* Este texto hace parte de mi investigación realizada en el marco de mi beca doctoral UBACyT, llevada a cabo en la Universidad de Buenos Aires, Facultad de Filosofía y Letras, Instituto de Historia Argentina y Americana "Dr. Emilio Ravignani”, financiada por la misma Universidad.

\section{Cómo citar este artículo:}

Alzu, A. N. (2020). Consideraciones para una actualización del concepto de totalidad social en base a la obra de Georg Lukács. Estudios de Filosofía, 62, 75-96. https://doi.org/10.17533/udea.ef.n62a05 


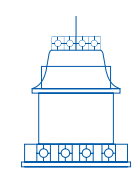

ARTÍCULOS

DE INVESTIGACIÓN

\title{
Considerations for an update of the concept of social totality based upon the work of Georg Lukács
}

\begin{abstract}
In this paper I revisit the different elaborations of the concept of social totality that we can find through various moments of the work of the Marxist philosopher Georg Lukács, focusing on the understanding of the praxis of social agents tied to each of them. I begin with the analysis of this concept in his youth works and then I highlight a radical change of view between the characterizations found in History and class consciousness and Ontology of social being. My argument is that this reconsideration is associated with the theoretical necessity of conceiving a subjective praxis that could avoid alienation, as it seems to occur in the theoretical model of Lukács' youth work. Finally, I conclude that this new conception of the social totality enables a new kind of emancipatory praxis, far from the party dogmatisms that typify a great part of the twentieth century socialism.

Palabras clave: Totality, reification, alienation, capitalism, Marxism
\end{abstract}

\section{Alejandro Nahuel Alzu}

Doctorando en Filosofía en la Universidad de Buenos Aires y becario doctoral UBACyT. Maestrando en Filosofía Política en la Universidad de Buenos Aires. Profesor de Enseñanza Media y Superior en Filosofía por la misma casa de estudios. Miembro del "Grupo de estudios sobre Marx y el Marxismo" del Instituto de Filosofía "Dr. Alejandro Korn” y de diversos grupos de investigación relacionados al marxismo y teoría crítica. 


\section{Introducción}

Sin lugar a duda, el filósofo marxista Georg Lukács es reconocido como uno de los padres del marxismo occidental (Anderson, 1979). En su obra Historia y conciencia de clase conceptualizó, mediante el término "reificación" (Verdinglichung), uno de los fenómenos sociales característicos de la vida moderna y se constituyó así en una influencia fundamental para los diversos desarrollos posteriores del marxismo. Sin embargo, el viraje intelectual que experimentó a partir de la década de 1930 le generó un fuerte rechazo a las posturas que allí expresaba. Entre las principales críticas que esgrime contra su propia obra se destaca el carácter totalizante que en esta tiene el concepto de reificación. Ante esta situación, pareciera que es la imposibilidad de concebir una práctica social no alienada dentro de aquel marco teórico lo que conduce a Lukács a desarrollar una nueva concepción de totalidad en sus obras posteriores, ahora desde una perspectiva ontológico-genética.

En este contexto, el presente trabajo tiene como objetivo mostrar el lugar que la totalidad social ocupa en la obra lukácsiana, particularmente en dos grandes momentos de esta: un primer momento representado por su obra de 1923, Historia y conciencia de clase (de aquí en más HCC), y un segundo momento representado por su obra póstuma Para una ontología del ser social, publicada en 1981. En este sentido, me propongo a explorar las continuidades y divergencias entre ambas obras para, con base en ello, indagar las consecuencias teóricas que este cambio de enfoque acarrea a la hora de pensar la posibilidad de una práctica social emancipatoria en la sociedad capitalista en el contexto de la obra lukácsiana, así como también para la elaboración de una teoría crítica de la modernidad adecuada a la realidad contemporánea.

Dado que para adentrarnos en la comparación de estas dos grandes obras sería óptimo una reconstrucción de diversos escritos previos del autor donde aparecen sus ideas principales, mi propuesta es, en primer lugar, reconstruir la concepción de totalidad que podemos encontrar en los primeros escritos de Lukács para mostrar cómo se decanta en las elaboraciones de la obra de 1923, haciendo hincapié en el lugar que ocupan el ser social y el actuar de los individuos dentro de esta formación. Luego, presentaré la autocrítica que Lukács hace de esta obra, para intentar comprender los fundamentos de este cambio de postura y para, en el siguiente apartado, presentar la concepción de totalidad que opera en su obra tardía y hacer foco en el lugar que ocupa la acción subjetiva en este último modelo teórico. Finalmente, evaluaré las consecuencias teórico-políticas que este cambio de perspectiva acarrea, así como también indagaré sobre el tipo de práctica emancipatoria que se puede llegar a concebir a partir de este nuevo marco teórico. 


\section{El concepto de totalidad en los escritos del joven Lukács: primeras apariciones del concepto}

Antes de comenzar a exponer la función del concepto de totalidad en HCC, vale la pena detenernos en los desarrollos previos que de este concepto podemos encontrar en la obra lukácsiana; pues, como plantea Martin Jay, para comprender correctamente el pensamiento del "teórico que colocó la categoría de totalidad en el corazón del marxismo occidental" (Jay, 1984, p. 84), debemos comenzar por analizar el funcionamiento de esta categoría en los trabajos de Lukács previos a su conversión al marxismo. ${ }^{1}$ Ante esta situación, cabe destacar que, si bien los ensayos que componen su primer libro, El Alma y las formas (1911), están dedicados al análisis estético y filosófico de la obra de diversos pensadores y artistas de preeminencia en la cultura austrohúngara de principios del siglo XX -entre los que se destacan Novalis, Stefan Georg y Kierkegaard-, podemos encontrar en ellos un diagnóstico romántico de la modernidad que se corresponde con formulaciones de su posterior período marxista. A los diversos ensayos que componen esta obra subyace la idea de que la vida es esencialmente caótica, que en su riqueza lucha constantemente por alcanzar una determinada forma que le dé coherencia, esto es, una cierta "totalidad normativa"; sin embargo, esa misma imposición de forma fracasa, pues en la modernidad todo intento de aprehensión de estas formas se convierte en la pérdida de lo que hace rica -y precisamente "viva" - a la vida, y así solo se termina por alcanzar una aprehensión de formas muertas. Según Lukács, este fenómeno no sólo se encuentra en las expresiones artísticas de los dos últimos siglos, sino que se refleja también en el ámbito de la vida cotidiana, pues impide tanto una auténtica relación de los individuos con su entorno como un adecuado establecimiento de relaciones interpersonales: "todo el mundo está tan cerca de nosotros que su proximidad transforma lo que nosotros le damos, y al mismo tiempo está tan lejos que en el camino entre los dos se extravía todo" (Lukács, 1985, p. 146). Y, si bien el joven Lukács expresa el anhelo de una totalidad de sentido originaria, anterior a la modernidad, ${ }^{2}$ esa totalidad se presenta como irrecuperable hoy en día; de modo que el hiato entre los hechos y nuestra comprensión de ellos se muestra irrecuperable:

Los hombres pueden entenderlo todo (...) pero esa comprensión no está ni puede estar en ninguna relación con lo realmente ocurrido. Desde el mundo del

1 Ante el estallido de la Revolución de octubre, hubo una verdadera conversión de un "romanticismo anticapitalista" al marxismo revolucionario por parte del pensador húngaro. Cf. Ios trabajos clásicos de Kadarkay, (1994), Arato \& Breines, (1979) y Löwy, (1979). Esta conversión, como plantea Lohmann (2012, p. 333), se caracterizó por una "fe incondicional” hacia el Comunismo que lo acompañó toda su vida, y que encontró expresión clara en su desprecio a los "compañeros de ruta" que sólo experimentaron una fascinación romántica y momentánea ante la Revolución rusa.

2 En palabras del autor: "Hubo tiempos — creemos que los hubo — en que esto que hoy llamamos forma y que buscamos con febril conciencia (...) era simplemente la lengua natural de la revelación, la libertad del grito, la energía inmediata de los agitados movimientos" (Lukács, 1985, p. 187). 
comprender (Verstehen) no se pueden echar más que vistazos al mundo de la vida; la puerta que conduce a este está eternamente cerrada, y ninguna fuerza del alma puede contribuir a volarla (Lukács, 1985, p. 185).

Frente a este diagnóstico romántico y pesimista de la modernidad, Lukács parece proponer como única actitud posible un escepticismo resignado: "nuestro conocimiento de los hombres es un nihilismo psicológico: vemos miles de relaciones y no captamos nunca una relación de conjunto" (Lukács, 1985, p. 146). Sin embargo, esta búsqueda de recuperar la totalidad de sentido perdida encuentra una propuesta positiva en su siguiente obra Teoría de la novela (1917), un estudio dedicado a establecer una historia de las formas literarias en el cual este concepto adquiere un carácter central en tanto opera como un "principio regulador abstracto" (Mészáros, 1981, p. 55) que es representado históricamente por la épica homérica. En esta obra el pensador húngaro plantea que el mundo griego de la épica de Homero "se trata de un mundo homogéneo, y [en él] aún la escisión entre hombre y mundo, entre 'yo' y 'tu' no perturba esa homogeneidad" (Lukács, 2010, p. 23); este mundo se presenta como una identidad originaria entre sujeto y objeto, en la cual "el alma se halla en el medio del universo, como cualquier otro elemento del sistema" (Lukács, 2010, p. 23). Dentro de esta totalidad cada acción del alma adquiere sentido, por esto, el hombre griego podía forjar relaciones con otros, que decantaban en estructuras que lo excedían y lo completaban, relaciones que se manifestaban en los lazos del amor, la familia y el Estado (Lukács, 2010, p. 19). Esta visión del mundo antiguo se contrapone al análisis lukácsiano de la época presente en la cual esa totalidad se ha roto:

El círculo cuya cerrada naturaleza constituía la esencia trascendental de su vida, para nosotros se ha fracturado. (...) Hemos hallado en nosotros mismos la verdadera y única sustancia, por eso establecemos un abismo insalvable entre el acto cognitivo y la acción, entre el alma y la figura, entre el ser y el mundo (Lukács, 2010, p. 25).

Esta "caída" se manifiesta en la cotidianeidad del hombre contemporáneo, en su constante búsqueda de esa totalidad perdida y sus repetidos fracasos, a la vez que la escisión entre las cosas y nuestra posible comprensión de ellas encuentra su máxima expresión en la fallida manera en que nos relacionamos con el mundo. Para desarrollar esta última cuestión, Lukács acuña el concepto de "segunda naturaleza", concepto que prefigura al de reificación (Verdinglichung), clave para comprender los aportes fundamentales de HCC al marxismo occidental. En este sentido, el joven Lukács plantea que el fenómeno de la falta de ligazón con ideales supra-personales de las estructuras sociales termina por constituir un complejo social definible por su opacidad de sentido, denominado "mundo de la convención" (Kovention):

Un mundo presente en todo lugar, en una multiplicidad de formas demasiado complejas para ser comprendidas. Sus estrictas leyes, tanto en el devenir como 
en el ser, son necesariamente evidentes para el sujeto cognoscente, pero a pesar de su regularidad, es un mundo que no se ofrece como sentido para el sujeto que busca fines, ni como sustancia, en inmediatez sensible, para el sujeto activo (Lukács, 2010, p. 56).

En el mundo de la convención, el sujeto se enfrenta a objetos, relaciones y estructuras cuyo sentido le resulta inaprensible, los cuales se presentan así como una "segunda naturaleza" que "como la primera, es determinable únicamente en tanto encarnación de necesidades reconocidas pero extrañas al sentido" (Lukács, 2010, p. 56). Sin embargo, esto no significa que las estructuras de la sociedad carezcan de sentido, tal como sí ocurre con la primera naturaleza "muda"; pues la segunda naturaleza es definida como "un complejo de sentidos - significados-que se ha vuelto rígido y extraño, y que ya no reaviva la interioridad; es un cementerio de interioridades en descomposición hace ya tiempo" (Lukács, 2010, p. 57). En este contexto, la única posible recuperación del sentido perdido en esta segunda naturaleza se da a través de un tipo de experiencia estética que permite un contacto inmediato con aquella totalidad armoniosa identificada con el mundo griego: "el acto metafísico que consiste en reavivar las almas que, en una existencia remota o ideal, la crearon o la preservaron" (Lukács, 2010, p. 57). Este acto no es otra cosa que la experiencia estética que se puede vivir mediante la lectura de una novela, única forma literaria que "busca descubrir y construir la totalidad de la vida oculta" (Lukács, 2010, p. 57). Sin entrar en mayores detalles sobre la exposición del autor que no competen a nuestro tema, me interesa particularmente destacar que en este texto la totalidad se vuelve a presentar vinculada a un acceso metafísico al sentido perdido de las relaciones sociales que constituye el diagnóstico de la modernidad; y, si bien esta totalidad de sentido a la que tiende la forma novela tiene un cierto anclaje histórico -expresado en la forma de la épica homérica-, no deja de ser una totalidad abstracta, un ideal regulativo de una experiencia estético-metafísica trascendente. Como veremos a continuación, el viraje marxista de Lukács luego de la Revolución rusa implicó una concreción en la concepción de la totalidad, que pasó de tener un sentido metafísico y trascendente a presentarse como una exigencia metodológica.

\section{La concreción de la totalidad: la reificación y el método dialéctico}

\subsection{La realización de la totalidad a través del socialismo}

El pasaje a una concepción concreta de la totalidad tiene un antecedente fuerte en un breve artículo llamado "Vieja y nueva Kultur" que Lukács publicó en 1919, mientras ejercía el cargo de Comisario de instrucción pública durante la breve República soviética de Hungría presidida por Béla Kun (Raddatz, 1975, p. 31). En él, se parte de la contraposición de dos conceptos clave de la sociología alemana contemporánea: Kultur y Zivilisation. Mientras esta última se refiere al dominio exterior del hombre sobre la 
naturaleza (Lukács, 1973, p. 83), la Kultur es entendida como el dominio interior del hombre, que comprende "el conjunto de actitudes y de productos dotados de valor que resultan superfluos en relación al inmediato sustento" (Lukács, 1973, p. 74), por lo que aparece solamente en las sociedades en las que las necesidades primarias han sido satisfechas. Ahora bien, mientras esta forma de praxis representa un desarrollo continuo, lento y orgánico en la que cada producto surge de un proceso unitario y acabado cuyo fin es expresar su valor de por sí (Lukács, 1973, p. 76), la Zivilisation en su forma más evolucionada -el capitalismo- ha instaurado una ruptura en esa continuidad. En la modernidad capitalista se torna imposible toda construcción de Kultur, pues en ella no existe clase que, por su ubicación productiva, esté llamada a su creación: hasta la clase dominante, la burguesía, se encuentra sometida al proceso de producción (Lukács, 1973, p. 75). Por otro lado, prefigurando cada vez más el concepto de reificación, Lukács plantea que este proceso encuentra su máxima expresión en la producción mercantil: "cada producto de la época capitalista, como también todas las energías de los productores y de los creadores, reviste la forma de mercancía (...) tiene valor sólo en cuanto mercancía vendible o adquirible en el mercado" (Lukács, 1973, p. 76).

Consecuentemente, la conversión de la sociedad en su conjunto en un "enorme mercado" (Lukács, 1973, p. 76) y la transformación de la vida social en una gran relación de intercambio hacen imposible la recuperación de ese "valor de por si" propio de los productos de la auténtica Kultur. De esta manera, la economía se torna un fin en sí misma: se torna "un proceso autónomo, dotado de legalidad propia" que puede ser reconocido mas no guiado por la razón humana (Lukács, 1973, p. 81) a la vez que el hombre entra en una relación de servilismo con ella.

Frente a esta totalidad social guiada por el "devenir-todo-mercancía" (Lukács, 1973, p. 81) el joven Lukács plantea que sólo el comunismo puede generar las condiciones para recuperar esa organicidad que implica la Kultur, al recuperar el presupuesto sociológico por el cual el hombre es un fin en sí. Este proceso se dará en dos sentidos. En primer lugar, el comunismo crea las condiciones económicas que permiten que cada uno tenga el modo de vida "que en la época precapitalista solamente las clases dominantes podían llevar", es decir, que no necesite agotar todas sus fuerzas vitales y energías para satisfacer sus necesidades primarias (Lukács, 1973, p. 75). En segundo lugar, la crítica socialista a la sociedad capitalista es capaz de hacer un develamiento de la totalidad, de la globalidad del proceso económico, y establecer así las bases para que la crítica de la totalidad se convierta en acción práctica en dirección de su transformación (Lukács, 1973, p. 82).

Podemos ver así cómo, en el artículo presentado, la totalidad encuentra dos expresiones. Un primer sentido negativo, la totalidad social que constituye el mercado, en la que impera "el abstracto preocuparse de la compra-venta" (Lukács, 1973, p. 84) y en la cual se rompen todos vínculos de continuidad; y un segundo sentido afirmativo, reflejado en el proceso unitario, orgánico, que constituye la Kultur a recuperar en la sociedad comunista cuyo fin es la idea del desarrollo superior del hombre. Si bien el 
artículo propone la necesidad de un pasaje de una totalidad hacia la otra vinculada a la realización de la sociedad comunista, a pesar de reconocer que la transformación económica implicada por el socialismo es un presupuesto para ello, este proceso de transformación social no deja de ser principalmente cultural; de hecho, retoma explícitamente la herencia del idealismo alemán a través de la propuesta de realización de la idea del hombre como fin en sí mismo (Lukács, 1973. p. 86). En contraste, veremos que la totalidad como concreción histórica va a ocupar un lugar central a partir de la obra que analizaremos a continuación: Historia y conciencia de clase.

\subsection{La totalidad en Historia y conciencia de clase}

Los diversos ensayos que componen esta obra de 1923 parten del supuesto según el cual el núcleo central que distingue al materialismo histórico de la ideología burguesa es el método dialéctico a través del que se logra, por un lado, "desprender los fenómenos de la forma inmediata en que se dan, hallar las mediaciones por las cuales pueden referirse a su núcleo, a su esencia, y comprender ese núcleo", y por otro lado, "conseguir comprensión de su carácter fenoménico, de su apariencia como forma necesaria de manifestarse" (Lukács, 2013a, p. 98). A partir de este método, la primera obra propiamente marxista de Lukács hace un diagnóstico de la sociedad capitalista según el cual las tendencias de su desarrollo muestran un proceso de abstracción y racionalización fundado en la división del trabajo, en el que la forma mercancía "influye decisivamente en la forma de la objetividad tanto de los objetos cuanto de los sujetos de la sociedad" (Lukács, 2013a, p. 193). En consecuencia, en primer lugar, "surge un mundo de cosas y relaciones cósicas cristalizado (el mundo de las mercancías)" en el cual el hombre "se enfrenta con su propia actividad, con su propio trabajo, como algo objetivo, independiente de él" (Lukács, 2013a, p. 191). Por otro lado, en segundo lugar, en este mundo de las mercancías se constituyen sistemas parciales aislados cuyas leyes se le contraponen al hombre como poderes invencibles y autónomos. Frente a esta situación, el método dialéctico consiste en el develamiento del verdadero carácter de la reificación como una apariencia necesaria -históricamente necesaria, consecuencia de su génesis en el terreno de la sociedad capitalista- a través de su vinculación con la "totalidad concreta" (Lukács, 2013a, p. 99). En palabras del autor: "La dialéctica (...) frente a esos hechos y esos sistemas parciales aislados y aisladores subraya la concreta unidad del todo, y descubre que esa apariencia es precisamente una apariencia" (Lukács, 2013a, p. 96). De esta manera, podemos ver que para Lukács tanto la forma de objetividad como la cognoscibilidad de un objeto están siempre definidas por "su función en la totalidad determinada en la que funcionan", por este motivo, sólo la consideración dialéctica de la totalidad es capaz de concebir la realidad como acaecer social (Lukács, 2013a, p. 106) y reconocer sus contradicciones como históricamente necesarias, con el fin de vislumbrar así las tendencias reales del proceso de desarrollo social Ilamadas a superarlas (Lukács, 2013a, p. 101). 
El concepto de totalidad opera entonces en $\mathrm{HCC}$ en un doble aspecto: ontológico y metodológico (Riu, 1968, p. 22). A la vez que es una categoría central del método dialéctico y así permite alcanzar el conocimiento de las relaciones entre las diversas formas objetivas de la realidad, también se manifiesta como totalidad concreta en la existencia histórica de la sociedad capitalista contemporánea, como el todo dialécticodinámico que contiene los diversos momentos que son las formas de objetividad (Lukács, 2013a, p. 104). Ahora bien, a este concepto de totalidad concreta podemos contraponerle el concepto de la reificación como forma de la totalidad abstracta que encontramos en la inmediatez del "mundo de las mercancías", que oculta el acaecer y las relaciones sociales tras las cosas. Podemos plantear así un tercer sentido de la totalidad operante en el texto, una totalidad abstracta, que se presenta como una discontinuidad de fragmentos que sólo pueden llegar a conformar "subsistemas" parciales, independientes e inconexos entre sí pero que presentan una estructura análoga (Jung, 2015, p. 22). Respecto a este último sentido de la totalidad, representada por el fenómeno de la reificación y su alcance a todas las esferas de la vida humana en la sociedad capitalista, la captación de la totalidad concreta que subyace a esta apariencia -el "desgarramiento de ese velo" (Lukács, 2013a, p. 104)- aparece como un acto de conocimiento, un proceso de "toma de conciencia", que sólo es posible en el capitalismo, dado que recién en esta época histórica "la sociedad llega a ser la realidad para el hombre" (Lukács, 2013a, p. 113). La particularidad de este proceso cognoscitivo es que a través de él se realiza la unidad del sujeto y objeto que colma el hiato entre la teoría y la praxis. ${ }^{3}$ El filósofo húngaro plantea que hay "una toma de conciencia del hombre acerca de sí mismo como ser social, acerca del hombre como sujeto y simultáneamente objeto del acaecer histórico social" (Lukács, 2013a, p. 113); sin embargo, esta toma de consciencia no puede llevarse a cabo por cualquier sujeto: mientras que la burguesía tiene un pensamiento "esencialmente antihistórico" (Lukács, 2013a, p. 281) y los intereses de clase la mantienen necesariamente presa de aquella inmediatez, opuestamente, el proletariado se encuentra empujado a rebasarla pues "sus formas de existencia (...) son de tal naturaleza que la cosificación tiene que mostrarse precisamente en ellas del modo más cargado y penetrante, produciendo la deshumanización más completa" (Lukács, 2013a, p. 272). Para Lukács, la situación cotidiana del trabajador, en la cual "se ve obligado a sufrir su conversión en mercancía" (Lukács, 2013a, p. 22) al vender su fuerza de trabajo, muestra cómo este se presenta en su situación inmediata como un mero producto del orden social capitalista; no obstante, esta misma situación genera una escisión en su ser social, entre su fuerza de trabajo y su personalidad toda, entre objetividad y subjetividad, que la torna así susceptible de

3 Precisamente en este sentido opera la dialéctica que Lukács busca recuperar para el marxismo. Esta recuperación de Hegel llevó a diversos autores a plantear que HCC debe ser entendido como una "fenomenología", ya sea en términos de una "ciencia de la experiencia de la conciencia”, en contraposición a una lógica o a una ontología (Infranca, 2005, p. 270), o incluso como una reformulación de la concepción husserliana a la hora de pensar la relación sujeto-objeto, aplicada a la sociedad capitalista (Westermann, 2019). 
conciencia (Lukács, 2013a, p. 294). Justamente ahí radica el corazón de la dialéctica del joven Lukács pues este autoconocimiento del trabajador es esencialmente algo práctico, en tanto consuma una alteración en la estructura objetiva de su objeto (Lukács, 2013a, p. 295) y se presenta, así, como el primer paso para romper el velo de la reificación de la totalidad social abstracta y revelar el carácter concreto de las relaciones sociales que se esconden tras ella.

Sin embargo, en este punto surge la cuestión de la posibilidad de alcanzar un conocimiento de la totalidad concreta a partir de nuestra existencia inmediata en la totalidad abstracta regida por la forma mercancía. Si en la vida cotidiana del ser social, aún más del trabajador, impera la reificación como "forma necesaria" de la realidad cubriendo todas las relaciones sociales, ¿cómo puede llegar a "tomar conciencia" de su situación y así de la totalidad concreta? Lukács reconoce que poder captar el sentido de esta totalidad "presupone una conciencia altamente desarrollada del proletariado acerca de su situación" (Lukács, 2013a, p. 116), es decir, no se puede corresponder con su conciencia empíricamente dada, tal como la encontramos en la realidad inmediata; por este motivo, el filósofo húngaro plantea la necesidad de la mediación del Partido Comunista para "elevar" esa consciencia inmediata a su "posibilidad objetiva", ${ }_{4}^{4}$ es decir, aquella consciencia "que tendrían los hombres en una determinada situación vital si fueran capaces de captar completamente esa situación y los intereses resultantes de ella" de "referir la conciencia al todo de la sociedad" (Lukács, 2013a, p. 148).

Cabe destacar que la concepción del partido que encontramos en HCC es fuertemente influida por la polémica entre las dos principales posturas de la literatura marxista acerca del "problema de la organización", es decir, de la relación entre el partido y las masas. Nos referimos, claro está, al debate entablado entre Lenin y Rosa Luxemburgo. En líneas generales, la postura representada por Lenin, que podemos encontrar claramente desarrollada en ¿Qué hacer? (1902), plantea la necesidad de una organización partidaria guiada por un restringido y estable grupo de dirigentes "que encaren las actividades revolucionarias como una profesión" (Lenin, 2013, p. 157) y así puedan guiar a las masas hacia la revolución socialista. Subyace a esta concepción la idea de que la mera "espontaneidad" del movimiento obrero -es decir, su desarrollo exclusivamente por sus propias fuerzas- es solamente la forma embrionaria de la conciencia revolucionaria, pues, a juicio del joven Lenin, ${ }^{5}$ el movimiento obrero librado a sí mismo es incapaz de ir más allá de una conciencia sindical que luche principalmente por los intereses económicos de la clase trabajadora. De este modo, un movimiento como

4 Sin embargo, como señala Jaffe (2020, p. 11), la remisión al partido como mediador que logra salvar el hiato entre la conciencia empírica y la conciencia atribuida, pareciera ser la remisión a una mera idea, pues en HCC no encontramos una determinación sociohistórica que caracterice el contenido de dicha conciencia imputada.

5 Es importante destacar que, como señala Tamás Krausz (2015, p. 115), la idea de que bajo un régimen burgués es imposible para los trabajadores alcanzar una conciencia socialdemocrática es revisada y abandonada por Lenin cinco años después de la publicación de ¿Qué hacer?, como consecuencia de las primeras revoluciones rusas de 1905. 
el socialismo requiere de la dirigencia partidaria de un grupo reducido de intelectuales revolucionarios que actúe de guía de todo el movimiento; en palabras de Lenin, la historia muestra que la conciencia socialdemócrata "sólo podía ser introducida desde afuera" (Lenin, 2013, p. 90). Frente a esta concepción de la organización, Rosa Luxemburgo considera que esta postura representa una tendencia "ultracentralista", que se basa en la separación drástica de los grupos organizados de revolucionarios activos de su medio ambiente, acompañada de la disciplina más estricta y la intervención directa de la autoridad central en todas las actividades de los grupos partidarios locales (Luxemburgo, 2013, p. 190). A diferencia de la postura leninista, Luxemburgo considera que la conciencia revolucionaria nunca puede ser introducida exteriormente, sino que surge en el proceso dialéctico de la lucha de clases. Como plantea la fundadora de la Liga Espartaquista: "La organización, los procesos de la conciencia y la lucha no son fases particulares, separadas mecánicamente en el tiempo (...) sino por el contrario son aspectos distintos de un mismo y único proceso" (Luxemburgo, 2013, p. 193). En este sentido, el espontaneísmo de Luxemburgo plantea que sólo al liberarse de la obediencia a un poder centralizado -ya sea el de un Estado burgués o su equivalente de un comité central socialista- para realizar sus actividades revolucionarias autónomamente, la clase obrera podrá adquirir la autodisciplina libremente consentida que caracteriza a una verdadera socialdemocracia (Luxemburgo, 2013, p. 194). En este contexto, la concepción del partido planteada en HCC viene a ofrecer una síntesis entre ambas posturas: mientras Lukács por un lado reconoce la importancia de la disciplina y el compromiso de todos los miembros en la organización partidaria, que presupone "la intervención activa de la entera personalidad" de sus miembros en esta tarea (Lukács, 2013a, p. 455), por otro lado, remarca la necesidad de tomar distancia de la organización de los partidos burgueses, en los que se pueden distinguir un núcleo de personas en cuyas manos se encuentra la dirección activa y el resto de los miembros de la asociación, que representan la función de objeto, en la forma de una masa pasiva. Por este motivo, introduce Lukács un segundo elemento de carácter espontaneísta: la necesidad de la intervención activa de todos los miembros del partido en su dirección bajo la forma de la autocrítica:

precisamente porque toda decisión del partido tiene que realizarse en las acciones de todos sus miembros (...) en las cuales éstos ponen en juego toda su existencia física y moral, los miembros están en situación de y están incluso, obligados a empezar inmediatamente su crítica, a formular inmediatamente sus experiencias, sus reservas, etc. (Lukács, 2013a, p. 474).

Esta necesidad de autocrítica de la forma partidaria que expresa su lucha consigo misma contra toda posible fetichización (Eiden-Offe, 2015, p. 92) termina de caracterizar la concepción del partido de HCC como el producto de una tensión entre el espontaneísmo luxemburguista y la centralización leninista. Ahora bien, esta tensión parece encontrar finalmente una resolución hacia la centralización, pues, 
en último término, el eslabón del Partido actúa en HCC como garantía del acceso al conocimiento del todo concreto, en tanto en su interior hay una vanguardia consciente que aparece como un "resto" inabarcable por la totalidad abstracta que representa la reificación. Así, como señala Löwy (2011), ya en su repuesta a dos reseñas negativas de HCC presumiblemente escrita en 1925, Lukács retoma a Luxemburgo, pero ahora como representante de "un modo de ver inmediatista y mitológico"6 (Lukács, 2015, p. 51) al que contrapone como referencia positiva una lectura mecanicista de la propuesta del joven Lenin acerca de la necesidad de introducir "desde afuera" la adecuada conciencia de clase. De esta manera, el desgarro de esa totalidad negativa que es la totalidad reificada sólo puede darse gracias a esta remisión al exterior que, si bien plantea la necesidad de una imbricación dialéctica con la conciencia existente de la clase trabajadora, no deja precisamente de provenir del exterior. En este sentido, ante la incapacidad de los sujetos de actuar en un sentido emancipatorio que sea inmanente a la realidad inmediata reificada, la remisión al exterior, mediante la remisión al partido con una vanguardia consciente, resulta la única posibilidad que habilita no sólo una revolución socialista, sino incluso cualquier acción conscientemente orientada hacia la emancipación social.

\section{Hacia un nuevo concepto de totalidad}

\subsection{El "viraje ontológico" de Lukács: los fundamentos para una ontología del ser social}

A partir de la década del 1930 Lukács comienza un período fuertemente autocrítico de su pensamiento anterior. Luego de la lectura de los entonces inéditos Manuscritos económico-filosóficos de Marx entre los años 1929 y 1930, a los que tuvo acceso mientras trabajaba en el Instituto Marx-Engels de Moscú, el pensador húngaro se encaminó a hacer una reelaboración de su pensamiento, reelaboración que se desarrollará constantemente a lo largo de su vida hasta llegar a su obra póstuma Para una ontología del ser social. Parte de este cambio de enfoque se refleja con claridad en el prólogo que escribe en 1967 a la segunda edición de HCC, el cual se destaca por su tono fuertemente crítico frente a la obra que introduce. Allí Lukács plantea que uno de los principales errores de esta obra fue no haber considerado el trabajo según una concepción propiamente marxista, es decir, "en cuanto mediador del intercambio de la sociedad con la naturaleza" (Lukács, 2013a, p. 54). A su juicio, su obra de juventud tenía una concepción idealista del trabajo fuertemente influida por la Fenomenología del espíritu hegeliana, incapaz de responder a la diferenciación que el joven Marx

6 Como muestra Clark (2018, p. 191), también en HCC hay numerosas críticas a Rosa Luxemburgo que tienden a exagerar su espontaneísmo; sin embargo, en esta obra estas coexisten con valoraciones positivas hacia su pensamiento que contrastan con la valoración de ella únicamente negativa que encontramos en Derrotismo y dialéctica. 
estableció entre objetivación (Vergegenständlichung) y alienación (Entfremdung), es decir, entre la materialización o fijación de todo trabajo humano en un objeto (Marx, 2010, p. 106) y su materialización como forma alienada dentro del capitalismo. De este modo, la alienación se presentaría en toda posición de objetividad u objetivación, es decir, toda producción de un objeto y toda acción serían indefectiblemente la realización de una forma alienada. Destaca Lukács, a su vez, que tal caracterización no es compatible con una concepción auténticamente materialista pues recae inevitablemente en el idealismo, ya que dentro de aquel marco teórico la superación de la alienación conllevaría necesariamente a la superación de toda objetividad. En palabras del autor:

como el objeto, la cosa, no existe según Hegel sino como la enajenación ${ }^{7}$ (Entäusserung) de la autoconciencia, ocurre que la retrocapción de la alienación en el sujeto sería el final de la realidad externa, o sea el final de la realidad en general (Lukács, 2013a, p. 61).

De esta manera, esta postura tiene que resolverse inevitablemente en un idealismo: en el espíritu absoluto que es sujeto-objeto autoconsciente de su propio despliegue en la historia en el caso de Hegel, o en la idéntica caracterización del proletariado en HCC. Una segunda consecuencia que nota Lukács es la cercanía de su posición a la idea de que la alienación es una condición humana (Lukács, 2013a, p. 62), pues la falta de distinción entre estos conceptos en términos filosóficos lo acercan peligrosamente a posturas existencialistas de las que buscó distinguirse durante toda su vida. Finalmente, una tercera consecuencia de este cambio de perspectiva es, como veremos en el siguiente apartado, una nueva manera de concebir la totalidad social en su obra de madurez.

Antes de entrar en la cuestión de la totalidad en Para una ontología del ser social, cabe hacer un par de aclaraciones sobre las circunstancias de publicación de dicha obra. Durante los últimos 15 años de su vida, Lukács se embarca en un magno proyecto de refundación teórica del marxismo conformado por tres grandes ramas: una Estética, una Ontología y una Ética. Cada una de ellas implicaba a la vez el proyecto de una obra independiente; sin embargo, el autor sólo llegó a publicar en vida la primera parte de su Estética, mientras que de la Ética sólo nos fueron legados fragmentos. Por otra parte, la Ontología -originariamente considerada una introducción a la Ética- estaba prácticamente concluida al momento de la muerte del autor y, si bien faltaban una serie de revisiones finales, esto no impidió que se publicara póstumamente en forma íntegra en 1984. Sabido esto, debemos tener siempre presente que esta obra es un análisis crítico de las formas del ser social desde una perspectiva ontológico-genética con el fin de plantear las bases de una praxis emancipatoria fundada en una ética no abstracta, que considere al ser humano como el centro de su interés (Thompson, 2011, p. 239).

7 Modifico la traducción de Manuel Sacristán del término Entäusserung para distinguirla de alienación (Entfremdung). 
Para comenzar, cabe preguntarnos qué entiende Lukács por "ontología". Al respecto, el pensador húngaro plantea que "no existe el ser en sentido estricto", pues este "es un proceso de índole histórica" (Holz, 1921, p. 26) y lo que cotidianamente denominamos así es "una fijación determinada y sumamente relativa de complejos dentro de un proceso histórico" (Holz, 1921, p. 26). Así, el acceso privilegiado a esta ontología es precisamente la vida cotidiana, el "punto gravitacional secreto" (Jung, 2001, p. 115) de la filosofía del pensamiento tardío de Lukács; particularmente el trabajo, entendido como la interrelación que se establece entre el hombre (la sociedad) y la naturaleza, se presenta ahora como aquel fenómeno originario en el cual "se hayan contenidas in nuce todas las determinaciones que (...) constituyen la esencia del ser social" (Lukács, 2004, p. 59). El trabajo es entonces el modelo de las determinaciones de este nuevo nivel ontológico que surge sobre la base del ser orgánico pero que es nuevo, distinto, más complejo: el ser social. Particularmente, dentro de las actividades del ser social, Lukács destaca la posición teleológica que requiere todo trabajo antes de ser llevado a cabo, es decir, "la postulación pensada de un fin" necesaria para "introducir en la realidad algo material que representa, frente a la naturaleza, algo cualitativamente y radicalmente nuevo" (Lukács, 2004, p. 69). Ahora bien, dado que dentro de la ideación previa al trabajo podemos distinguir la posición de fines y la investigación de los medios para la realización de esos fines, el filósofo húngaro destaca ambos momentos como los dos actos ontológicamente originarios de la esfera del ser social, cuya interacción expresa el vínculo indisoluble entre la teleología y la causalidad (Lukács, 2004, p. 70). Así, podemos afirmar que mientras en la naturaleza las cadenas causales se desarrollan "espontáneamente" según su necesidad natural del tipo "si... entonces" (Lukács, 2004, p. 90), el proceso del trabajo consiste en transformar la causalidad natural en causalidad puesta (Lukács, 2004, p. 73), es decir, adecuar esas cadenas causales hacia el fin propuesto.

Se va constituyendo así, por la acción del ser humano a lo largo de largos períodos de tiempo, una serie de complejos que comienzan a diferenciarse y a adquirir autonomía y que en su conjunto constituyen el "complejo de complejos" orientado a satisfacer las necesidades de la reproducción humana que es el ámbito propio del ser social. Debemos guardarnos aquí de comprender la conformación del complejo social como una mera replicación del proceso del trabajo, pues la dinámica propia del desarrollo del ser -la "identidad de la identidad y la no identidad" (Lukács, 2004, p. 115) - opera a través de saltos: cada nuevo nivel surge por el desarrollo de una mayor complejidad a partir de un nivel anterior, pero a la vez, adquiere una dinámica que lo torna independiente de aquel. ${ }^{8}$ Como plantea Lukács:

8 Así, por ejemplo, en las formas más evolucionadas de la praxis social aparece una segunda categoría de acto social: aquella acción orientada a fines que ya no es una mediación directa con la naturaleza, sino que su posición teleológica es "la tentativa para conseguir que un hombre (o un grupo de hombres) realice, por su parte, posiciones teleológicas concretas” (Lukács, 2004, p. 103). Este tipo de acción más compleja, que se propone actuar sobre un material "no indiferente" (Lukács, 2004, p. 112), puede ser leída como un segundo tipo de acción que se propone en la ontología lukácsiana (Maceo da Costa, 2012). 
cada salto significa una transformación cualitativa y estructural en el ser, en la cual el estado inicial contiene dentro de sí, sin duda, determinadas condiciones y posibilidades de la posterior y más elevada, pero estas no pueden ser desarrolladas a partir de aquellas según una continuidad simple y rectilínea (Lukács, 2004, p. 60).

Desde el punto de vista de los individuos que conforman ese complejo social, toda posición en el proceso de trabajo implica una elección; las personas se encuentran constantemente en su vida social en situaciones que implican una toma de decisión. Sin embargo, en los niveles más evolucionados de la sociedad, la alternativa a la que se enfrentan los individuos no es un acto único de decisión sino una "cadena atemporal ininterrumpida de alternativas siempre nuevas" (Lukács, 2004, p. 91), por lo que la estructura ontológica del proceso del trabajo se convierte en una "cadena de alternativas". Ahora bien, el pensador húngaro aclara que toda alternativa es una elección concreta entre caminos cuyo fin "no fue producido por el sujeto que decide, sino por el ser social en el que ese sujeto vive y actúa" (Lukács, 2004, p. 95), pues el complejo del ser social, que existe independientemente de los individuos particulares, determina siempre en última instancia la posición de las series causales sobre las que estos actúan: en la ontología de Lukács la estructura total del complejo es algo primario frente a sus elementos. Se introduce así, en el pensamiento tardío de Lukács, la categoría de totalidad social como un momento predominante, principalmente en dos aspectos: uno vinculado a su rol como mediación social que determina los elementos que pertenecen a cada complejo, y un segundo aspecto ligado al criterio epistemológico necesario para tener una correcta comprensión del ser social que pueda servir de fundamento para una praxis emancipatoria. Me avocaré al análisis de estos dos aspectos en el siguiente y último apartado.

\section{2. El lugar de la totalidad en Para una ontología del ser social}

Dentro de la ontología desarrollada por el filósofo húngaro, podemos encontrar una caracterización de la totalidad social donde se destacan dos funciones: la totalidad social como mediación concreta de todos los elementos que conforman el complejo de complejos que es la sociedad y la totalidad entendida como criterio epistemológico a través del cual abordar un análisis ontológico crítico de la sociedad contemporánea. Comencemos por el análisis de la primera función.

En primer lugar, debemos tener presente que toda posición teleológica que postula un individuo al actuar y particularmente al tomar una decisión frente a una alternativa está en última instancia determinada por el todo social, que establece "el ámbito de juego concretamente delimitado para las preguntas y respuestas posibles, para las alternativas que pueden ser realmente realizadas" (Lukács, 2004, p. 96). Con 
todo, no debemos olvidar que justamente esta totalidad se realiza, a su vez, a partir de las posiciones teleológicas individuales: "aún la más compleja economía es una resultante de posiciones teleológicas individuales, de las realizaciones de estas, ambas bajo la forma de alternativas" (Lukács, 2004, p. 137). Ahora bien, lo que ocurre es que el encadenamiento causal que generan dichas alternativas produce un movimiento social "cuyas determinaciones en última instancia se sintetizan en una totalidad procesual" que, a partir de cierto nivel de desarrollo de la sociedad, ya no puede ser captada por los individuos para orientar sus acciones (Lukács, 2004, p. 137). Este fenómeno aparece claramente en la modernidad capitalista, pues para Lukács los individuos ya no orientan sus acciones en base a la totalidad social, sino que existen determinaciones específicas de esta totalidad destinadas a conducir e influir en sus decisiones. Particularmente, en la sociedad contemporánea esto se manifiesta en "la división del trabajo mediada y puesta en marcha por el valor de cambio" que "produce el principio de dominio del tiempo a través de su mejor aprovechamiento intrínseco" (Lukács, 2004, p. 138). La totalidad social se presenta como mediación en este dominio del ámbito de los individuos por parte del tiempo, acompañado por un proceso de valorización que constituye así una "ley de la producción social", que actúa decisivamente sobre los actos individuales y genera una situación en la cual "el individuo debe adaptarse a esta ley, bajo amenaza de ruina" (Lukács, 2004, p. 138).

Cabe hacer dos aclaraciones sobre este primer aspecto de la totalidad social. En primer lugar, el filósofo húngaro reconoce que, a pesar de que esta demarca el ámbito de las decisiones de los individuos y los compele a decidir de una determinada manera, en toda alternativa hay un momento de decisión ejercido por la conciencia humana y allí radica precisamente el "núcleo ontológico de la libertad" (Lukács, 2004, p. 96), lo que distingue al ser social del ser biológico, al ser humano de los animales. Es fundamental no olvidar que más allá de las determinaciones impuestas por la totalidad, los individuos cuyas acciones encadenadas conforman esta totalidad están constantemente decidiendo entre alternativas. Y, en segundo lugar, Lukács destaca que este proceso de organización de la totalidad produce un ser social situado en un nivel superior de socialización, esto es, permite que la socialización se presente de una manera más pura (Lukács, 2004, p. 138). Esta situación debe ser entendida como un paso en el enriquecimiento del ser social, un paso hacia el "para sí de la socialización desarrollada" cuya encarnación adecuada es el propio ser humano, entendido como el individuo concreto que "en sus acciones encarna y hace realidad el género humano" (Lukács, 2004, p. 139). El mismo desarrollo histórico del ser social se presenta entonces como la posibilidad objetiva de su pleno desarrollo, pues posibilita el pasaje de la genericidad [Gattungsmäßigkeit] humana en sí a la genericidad para sí. ${ }^{9}$ Esto es,

9 Como plantea Lessa, "dada la radical historicidad del mundo de los hombres postulada por Lukács, luego de Marx (...) ninguna naturaleza humana podría, bajo ninguna hipótesis, constituirse en límite a priori para el desarrollo del devenir humano de los hombres" (Lessa, 2014, p. 121); es decir, la genericidad para sí no debe ser entendida como una esencia cerrada sino como un proceso dialéctico de desarrollo dinámico. 
permite el pasaje desde aquella genericidad que se expresa a partir del desarrollo del trabajo y se estructura para transformar ininterrumpidamente la vida inmediata de los hombres en vistas a cumplir las funciones del proceso de reproducción social, hacia un nuevo tipo de genericidad en la cual dejan de estar en contraposición el individuo y el género, es decir, en la cual se supera la alienación (Entfremdung). Pues este último concepto es entendido ontológicamente como el fenómeno por el cual el desarrollo de la sociedad y el despliegue de las capacidades humanas que implica "no acarrea necesariamente un desarrollo de la personalidad humana" sino que "por el contrario, (...) puede justamente mediante un despliegue de capacidades individuales distorsionar, degradar, etc. la personalidad humana" (Lukács, 2013b, p. 31).

Precisamente uno de los elementos clave para favorecer el desarrollo de las potencialidades del ser social es el conocimiento del rol mediador de la totalidad social, de que "todas las representaciones ontológicas de los hombres se encuentran (...) bajo la influencia de la sociedad" (Lukács, 2004, p. 117). De hecho, las necesidades sociales dominantes -representadas en el capitalismo contemporáneo por la "manipulación de la economía" (Lukács, 2004, p. 119) - se han convertido en un factor determinante en el proceso de reproducción social, al nivel de que han llegado a desarrollar una falsa conciencia ontológica en el campo de la ciencia ${ }^{10}$ que se expande a todos los campos de la praxis y se presenta como un verdadero obstáculo para alcanzar este conocimiento. Frente a esta situación, el filósofo húngaro postula la necesidad de elaborar una "crítica ontológica consciente" que, como tal, debe ser "necesariamente una crítica concreta, fundada en la totalidad social respectiva, orientada a la totalidad social" (Lukács, 2004, p. 120). Se revela así el segundo aspecto de la totalidad social que encontramos en la ontología lukácsiana: su función como criterio epistemológico para la correcta comprensión de los fenómenos sociales. En palabras del autor: "La crítica ontológica tiene que orientarse, pues, en dirección al todo diferenciado -concretamente diferenciado, en términos de clases- de la sociedad, y a las interrelaciones en los modos de comportamiento que de allí se derivan" (Lukács, 2004, p. 120).

Este tipo de conocimiento de la totalidad social en todas sus determinaciones -entre las que Lukács destaca las clases sociales- sólo es alcanzable a través del análisis ontológico marxiano que plantea el autor, a la vez que es un requisito indispensable para definir toda ética. Sólo a través de su conocimiento se puede llegar a orientar la praxis en un sentido emancipador y a superar la alienación para realizar la verdadera libertad humana, que no es otra cosa para el autor que contribuir a la realización de la genericidad humana para sí en cada acto individual: llevar a acto la capacidad del ser humano de "elevarse sobre la propia particularidad de una manera consciente" (Lukács, 2013b, p. 50).

10 Para Lukács, un ejemplo claro al respecto es el neopositivismo que propone el rechazo de toda investigación ontológica por considerarla metafísica, acientífica, pero que termina por caer en la postulación de la manipulación técnica y la creciente aplicabilidad práctica como telos de la metodología científica (Lukács, 1984, p. 314). 


\section{Conclusiones}

Hemos visto cómo la categoría de totalidad tiene una presencia fundamental durante todas las etapas del pensamiento de Georg Lukács, desde su romanticismo anticapitalista de los años previos a la Gran Guerra hasta su obra ontológica tardía, pasando por la obra que le dio una clara preeminencia a esta categoría en los futuros desarrollos del marxismo occidental, Historia y conciencia de clase. En primer lugar, se destaca en sus textos premarxistas, una concepción de la totalidad como una unidad originaria que en su momento fuera expresión de una relación armónica del ser humano con su entorno, tanto respecto a la sociedad como a la naturaleza; identificada en Teoría de la novela con la edad de oro de la Grecia de Homero. Se presenta así una totalidad entendida en un sentido positivo, que comienza a actuar como modelo o criterio de valoración del presente. Cabe destacar que, si bien tanto de El alma y las formas como de Teoría de la novela se desprende claramente un diagnóstico - profundamente negativo- de la modernidad capitalista, no dejan de ser textos que buscan analizar diversas formas literarias y construir, en el caso de la última obra, una historia de estas formas, cuyo criterio valorativo será esa totalidad homogénea entre el hombre y su entorno que fuera encarnada originalmente en un pasado remoto. Este sentido de la totalidad, que hasta ahora se presenta como un acceso metafísico al sentido perdido de las relaciones sociales, toma forma concreta hacia los primeros textos marxistas del filósofo húngaro, a la hora de plantear el conocimiento de la totalidad social como un criterio epistémico para llegar a comprender el verdadero sentido de las acciones y procesos sociales.

Por otro lado, ya en este primer momento aparece un segundo aspecto negativo de la totalidad ligado a la representación de la experiencia de la vida inmediata de los individuos, que encuentra su punto cúlmine en HCC. Así, la experiencia de ruptura entre los individuos y el sentido de las acciones sociales se manifiesta claramente en sus obras de juventud en la forma de la "tragedia de la cultura" (Feenberg, 2014, p. 62) -ya sea como una relación trunca entre una vida en constante cambio y los fallidos intentos del alma humana de aprehenderla, o como la imposibilidad del hombre por trascender ese carácter de "segunda naturaleza" que tienen los objetos sociales en la modernidad-; en este sentido, podemos afirmar que estas obras de juventud comparten con HCC el mismo punto de partida que es el carácter problemático de la existencia humana (Olay, 2016, p. 110). No obstante, en su obra de 1923 es claro que la solución a este problema adquiere mayor concreción, pues ya no se busca en la cultura una posible orientación que pueda dar sentido al sinsentido: la responsabilidad ética del héroe que en la forma novela luchaba por recuperar una trascendencia dentro de un mundo devenido inhumano se convierte en HCC en la misión profundamente ética del nuevo sujeto que es el proletariado: redimir a la humanidad a través de una revolución total del capitalismo (Charbonnier, 2016, p. 78). Con todo, bajo el velo de la reificación, la acción de los individuos se ve reducida a la mera contemplación de leyes cuasi-naturales propias del mecanismo del todo social, y estos no se presentan como dueños de su 
propio destino sino como espectadores del curso de los acontecimientos. Ahora bien, como vimos, ya en 1919, en su artículo sobre la Kultur, aparece en Lukács la idea de la totalidad como un criterio epistemológico para contrarrestar esta tendencia, idea que se desarrolla en detalle en HCC. Plantea entonces Lukács que las acciones y los procesos sociales sólo pueden ser entendidos adecuadamente si se llega a captar su función en la totalidad social a través del método dialéctico; la totalidad social, ahora concreta e históricamente definida, aparece como la mediación necesaria para llegar a tener una correcta comprensión de los procesos sociales y poder romper el velo de la reificación.

Sin embargo, la presencia plena de la reificación en la realidad inmediata conduce a que este conocimiento de la totalidad social se vuelva imposible para los individuos inmersos en la sociedad capitalista; incluso para la clase social empujada constantemente a rebasarla, el proletariado. Se necesita entonces una instancia sustraída a la reificación, que Lukács identifica con el Partido comunista, del que se destaca la vanguardia consciente que debe marcar la dirección de las acciones de la clase obrera para darles un sentido emancipatorio. De esta manera, el partido se revela como garantía última del acceso al conocimiento del todo concreto y también como un recurso necesario ante la imposibilidad de pensar una praxis social no alienada dentro de la sociedad capitalista. Se podría pensar que la figura del partido es la salida que propone Lukács frente a este diagnóstico pesimista que, llevado a su máxima expresión, recaería en posturas como la de Adorno al afirmar en su Dialéctica negativa que la única idea de historia universal viable para el ser humano inmerso en la reificación capitalista es una suerte de "teleología negativa" (Sotelo, 2009), de "avance ininterrumpido hacia el sufrimiento absoluto" (Adorno, 2005, p. 295).

Sin embargo, esta dependencia del partido puede generar consecuencias epistémicas sumamente problemáticas, tal como lo verificó Lukács ante la brutal manipulación estalinista (Lukács, 2013b, p. 251), pues delegar en el partido el rol de único garante de la posibilidad de un adecuado conocimiento de la sociedad para orientar correctamente la acción individual y colectiva le concede a éste una autonomía tal y plantea una subordinación de los agentes sociales a su institucionalización tan fuerte que fácilmente puede decantar en una manipulación. " Ante esta situación, no creo exagerado suponer que la lectura de los Manuscritos del joven Marx (2010) y su distinción entre objetivación y alienación, además de ayudar a develar el ya mencionado idealismo presente en HCC, fueron claves para ayudar a Lukács a plantear la posibilidad de una acción emancipatoria dentro de la totalidad social de la que inevitablemente todo agente forma parte, esto es, sin recurrir a una instancia externa. Considero que esto se puede ver reflejado en la Ontología, donde se abre la posibilidad de una praxis social no alienada dentro de las posibilidades de acción de los individuos. Como vimos, en esta obra el complejo de complejos que configura el ser social se va constituyendo a través de decisiones individuales entre alternativas que construyen cadenas de decisiones que, a su

11 Sobre las consecuencias totalizantes y autoritarias de esta fundamentación "exterior" del partido cf. Congdon, (2007) y Fraccia (2013). 
vez, conforman una causalidad puesta que se sintetiza en una totalidad. Esto no quita que el momento de la totalidad social tenga preeminencia frente a las partes -preeminencia que muchas veces se expresa en una imposición sobre los individuos con una coacción tal que estos se ven compelidos a decidir de una determinada manera- y que, por lo tanto, toda correcta comprensión de los elementos sociales implique comprender su lugar y su función en la totalidad que los determina. Sin embargo, debemos tener en cuenta dos cuestiones a la hora de pensar la totalidad social concebida por la ontología lukácsiana y el campo de posibilidades que los individuos tienen para actuar en ella. En primer lugar, el mismo desarrollo histórico del ser social se presenta como la posibilidad objetiva del pleno desarrollo del género humano, en tanto genera las condiciones materiales y culturales para el pasaje de la genericidad humana en sí a la genericidad para sí. Y, en segundo lugar, la ontología lukácsiana mantiene siempre abierta a los individuos la posibilidad de decidir entre alternativas; en este sentido, la ética se presenta una vez más como clave para la superación de la alienación y la emancipación del ser humano.

Para finalizar, considero que el viraje ontológico que le permitió a Lukács superar la dicotomía entre pesimismo y vanguardismo voluntarista que se desprende del planteo de su obra marxista de juventud puede ayudarnos también a pensar las posibilidades de acción emancipatoria inmanentes a la totalidad social de la que formamos parte, sin la necesidad de recurrir a instancias externas que por su total autonomía pueden resultar epistémicamente limitantes y políticamente peligrosas. Particularmente, estimo que una perspectiva ontológica que recupere en el sentido lukácsiano la articulación entre el obrar de los agentes sociales y la complejidad de la totalidad social puede servir para fundar las bases de una ética que permita orientar de manera realista -escapando tanto al determinismo como al utopismo- una acción colectiva en la lucha contra la desigualdad y las opresiones que se presentan en diversos espacios de la sociedad contemporánea. De esta manera podremos sacar provecho en un sentido liberador de las nuevas potencialidades que nos ofrece la modernidad capitalista, tanto para pensar una nueva relación con la naturaleza que vaya más allá del consumo y la depredación -tristemente común a nuestra actualidad orientada por el modo de producción capitalista y a los "socialismos realmente existentes" del siglo pasado-, como para repensar nuestros vínculos sociales más allá de la explotación y el sometimiento de la humanidad por la humanidad misma. Queda indagar para futuras investigaciones cómo una ética futura sobre un fundamento ontológico así delineado puede servir de guía para una praxis social emancipatoria, así como también qué tipo de acciones colectivas e individuales y qué organizaciones pueden llegar a seguirse de ella.

\section{Referencias}

Anderson, P. (1979). Consideraciones sobre el marxismo occidental. México: Siglo XXI.

Arato, A. y Breines, P. (1979). El joven Lukács y los orígenes del marxismo occidental. México: Fondo de cultura económica. 
Adorno, T. (2005). Dialéctica Negativa - La jerga de la autenticidad. Madrid: Akal.

Charbonnier, V. (2016). De Lukács à Lukács: itinéraire d`un remembrement. Romanesques: revue du Centre d'études du roman et du romanesque de l'Université de Picardie-Jules Verne (Lukács 2016: cent ans de Théorie du roman), 8, 175-186.

Clark, K. (2018). Rosa Luxemburg: The Russian Revolution. Studies in East European Thought, 70, 153-165. https://doi.org/10.1007/s11212-018-9305-5

Congdon, L. (2007). Apotheosizing the Party: Lukács Chvostismus und Dialectic. Studies in East European Thought, 59, 281-292. https://doi.org/10.1007/s11212-007-9039-2

Eiden-Offe, P. (2015). Kampf-Form. Versuch über die Form der Partei bei Georg Lukács. En H. Plass (Ed.). Klasse - Geschichte - Bewusstsein. Was bleibt von Georg Lukács' Theorie? (pp. 79-103). Berlin: Verbrecher Verlag.

Feenberg, A. (2014). The philosophy of Praxis. Marx, Lukács and the Frankfurt School. London: Verso.

Fracchia, J. (2013). The Philosophical Leninism and Eastern 'Western Marxism' of Georg Lukács. Historical Materialism: Research in Critical Marxist Theory, 21(1), 69-93. https://doi.org/10.1163/1569206X-12341282

Holz, H. (Ed.). (1971). Conversaciones con Lukács. Madrid: Alianza.

Infranca, A. (2005). Trabajo, individuo, historia. El concepto de trabajo en Lukács. Buenos Aires: Herramienta.

Jay, M. (1984). Marxism and Totality. California: University of California Press.

Jaffe, A. (2020). Lukács' antinomic 'standpoint of the proletariat': From philosophical to sociohistorical determination. Thesis Eleven, 157(1), 60-79. https://doi.org/10.1177/0725513620911841

Jung, W. (2015). Mercancía corpórea y cuerpo mercantil. El ensayo de Lukács sobre la cosificación. Revista Exlibris, 4, 18-32.

Jung, W. (2001). Von der Utopie zur Ontologie. Zehn Studien zu Georg Lukács. Bielefeld: Aisthesis Verlag.

Kadarkay, A. (1994). Georg Lukács: vida, pensamiento y política. Valencia: Alfons el Magnanim. Krausz, T. (2015). Reconstructing Lenin. An intellectual biography. Nueva York: Monthly Review Press.

Lenin, V. I. (2013). ¿Qué hacer? Problemas candentes de nuestro movimiento. En Obras selectas, Tomo I. Buenos Aires: Ediciones IPS.

Lessa, S. (2014). Para comprender la ontología de Lukács. La Plata: Editorial Dynamis.

Lohmann, H. M. (2012). Kommunismus als Religion. En M. Bitterolf \& D. Maier (Eds.). Verdinglichung, Marxismus, Geschichte. Vor der Niederlage der Novemberrevolution zur kritischen Theorie (pp. 331-337). Friburgo: Ça Ira.

Löwy, M. (2011). Revolutionary dialectics against 'Tailism': Lukács' answer to the Criticism of History and Class Consciousness. En M. J. Thompson (Ed.). Georg Lukács Reconsidered. Critical Essays in Politics, Philosophy and Aesthetics (pp. 65-72). Londres: Continuum. 
Löwy, M. (1976). Georg Lukács - From Romanticism to Bolshevism. Londres: NLB.

Lukács, G. (2015). Derrotismo y dialéctica. Una defensa de Historia y conciencia de clase. Buenos Aires: Herramienta.

Lukács, G. (2013a). Historia y conciencia de clase. Buenos Aires: Razón y revolución.

Lukács, G. (2013b). Ontología del ser social. La alienación. Buenos Aires: Herramienta.

Lukács, G. (2010). Teoría de la novela. Buenos Aires: Godot.

Lukács, G. (2005). Táctica y ética. En Táctica y ética. Escritos tempranos (1919-1929) Buenos Aires: El cielo por asalto.

Lukács, G. (2004). Ontología del ser social. El trabajo. Buenos Aires: Herramienta.

Lukács, G. (1985). El alma y las formas. Teoría de la novela. México: Grijalbo.

Lukács, G. (1984). Prolegomena. Zur Ontologie des gesellschaftlichen Seins, 1. Halbband. En Werke, Band 13. Darmstadt: Luchterhand.

Lukács, G. (1981). Gelebtes Denken. Eine Autobiographie im Dialog, Fráncfort del Meno: Suhrkamp.

Lukács, G. (1973). Vieja y nueva Kultur. En Revolución socialista y antiparlamentarismo (pp. 74-86). Buenos Aires: Pasado y Presente.

Luxemburgo, R. (2013). Problemas de organización de la socialdemocracia rusa. En Obras escogidas (pp. 188-205). México: Partido de los Trabajadores.

Maceo da Costa, G. (2012). Indivíduo e sociedade. Sobre a teoria de personalidade em Georg Lukács. São Paulo: Instituto Lukács.

Marx, K. (2010). Manuscritos económico-filosóficos. Buenos Aires: Colihue.

Mészáros, I. (1981). El pensamiento y la obra de G. Lukács. Barcelona: Fontamara.

Olay, C. (2016). Die Kulturtragödie menschlicher Existenz beim Lukács. En C. Lörincz (ed.). Wissen - Vermittlung - Moderne. Studien zu den ungarischen Geistes- und Kulturwissenschaften um 1900 (pp. 93-111). Köln: Böhlau. https://doi.org/10.7788/9783412218980-005

Raddatz, F. (1975). Georg Lukács. Madrid: Alianza.

Riu, F. (1968). Historia y totalidad. El concepto de reificación en Lukács. Caracas: Monte Ávila.

Sotelo, L. (2009). Ideas sobre la historia. La escuela de Frankfurt: Adorno, Horkheimer, Marcuse. Buenos Aires: Prometeo.

Thompson, M. J. (2011). Ontology and Totality: Reconstructing Lukács Concept of Critical Theory. En M. J. Thompson (Ed.). Georg Lukács Reconsidered. Critical Essays in Politics, Philosophy and Aesthetics (pp. 229-250). Londres: Continuum.

Westerman, R. (2019). Lukács's Phenomenology of Capitalism. Reification Revalued. Cham: Palmgrave Macmillan - Springer. https://doi.org/10.1007/978-3-319-93287-3 